\title{
Estimation du seuil anaérobie chez le poney
}

\author{
J.P. Valette, E. Barrey, C. Garbasi et R. Wolter \\ avec la collaboration technique de M. Jouglin
}

Ecole Nationale Vétérinaire d'Alfort, laboratoire de physiologie sportive INRA, 7, avenue du Général-de-Gaulle, 94704 Maisons-Alfort Cedex, France

(reçu le 20 février 1989; accepté le 12 juillet 1989)

Résumé - Chez le poney, le seuil anaérobie, état d'équilibre entre vitesses de production et de disparition de l'acide lactique dans le compartiment sanguin, est variable selon le type d'entraînement auquel l'animal est soumis. Par rapport à un exercice modéré (au pré), ce seuil s'abaisse en endurance (vers 2-2,5 mmol..$^{-1}$ ) et augmente en sprint (autour de 3,5 mmol...$^{-1}$ ). Ce seuil reste une valeur susceptible de fluctuations importantes dont la connaissance peut permettre l'orientation vers une discipline et un entraînement approprié.

seuil anaérobie - V4 - endurance - sprint - lactatémie

Summary - Estimation of anaerobic threshold in the pony - Six ponies were trained first for endurance for 6 months, then they were left for 2 months in a meadow and finally were trained for 5 weeks. They were tested on a treadmill to determine their anaerobic threshold $\left(L_{\text {seuil }}\right)$ and to compare it with the conventional 4 mmol.t-1 reference (Fig. 1) by incremental or constant speeds tests (Fig. 2).

It appears (Table I) that in the pony, the anaerobic threshold steady state between production and metabolization rate of lactic acid, varies according to animals and type of training. With endurance training, it decreases to $2-2.5 \mathrm{mmol}^{-1}$, while with speed training it increases up to 3.5 mmol.t-1.

The 4 mmol.t-1 value (Table II) does not seem to correspond to a threshold, because of the accumulation of lactic acid during prolonged exercice. The determination of the true threshold, which is an individual, variable value, is of importance in the management of a specific training.

anaeroblc threshold $-V_{4}$ - endurance - speed - blood lactate 


\section{INTRODUCTION}

L'appréciation de la valeur sportive d'un animal athlète tel que le cheval, nécessite une étude multifactorielle (Barrey et al., 1989) des paramètres susceptibles d'être influencés par l'entraînement, notamment les caractéristiques du métabolisme énergétique.

L'adaptation physiologique à un exercice d'endurance et de résistance, repose essentiellement sur le concept de seuil anaérobie et sur l'étude de la zone de transition aéro-anaérobie, décrits chez l'athlète humain par Wasserman et Mcllroy (1964) puis précisés par Mader et al. (1976), Kindermann et al. (1979) et Rieu et al. (1986).

Ce seuil correspond à un état d'équilibre entre les vitesses de production et de disparition de l'acide lactique dans le compartiment sanguin (Brooks, 1985). Cet équilibre serait permis, entre autres, par le couplage des fibres hypo- et hyperaérobies (Di Prampero, 1986). Au-delà de ce seuil, la lactatémie croît suivant un modèle exponentiel (Fig. 1), la couverture des besoins énergétiques se faisant alors majoritairement par voie anaérobie, parallèlement à un recrutement croissant des fibres à contraction rapide.

Cette notion de seuil anaérobie, situé chez l'athlète humain à $4 \pm 1$ mmol...$^{-1}$ (Mader et al., 1976) a donné lieu, selon les disciplines et les méthodologies d'évaluation à des définitions et déterminations particulières (Hollman, 1985) telles que I'IAT (Individual Anaerobic Threshold) de Keul et al. (1979) et l'OBLA (Onset of Blood Lactate Accumulation) de Sjodin et Jacobs (1981). La référence $4 \mathrm{mmol}^{-1} \mathrm{I}^{-1}$, justifiée

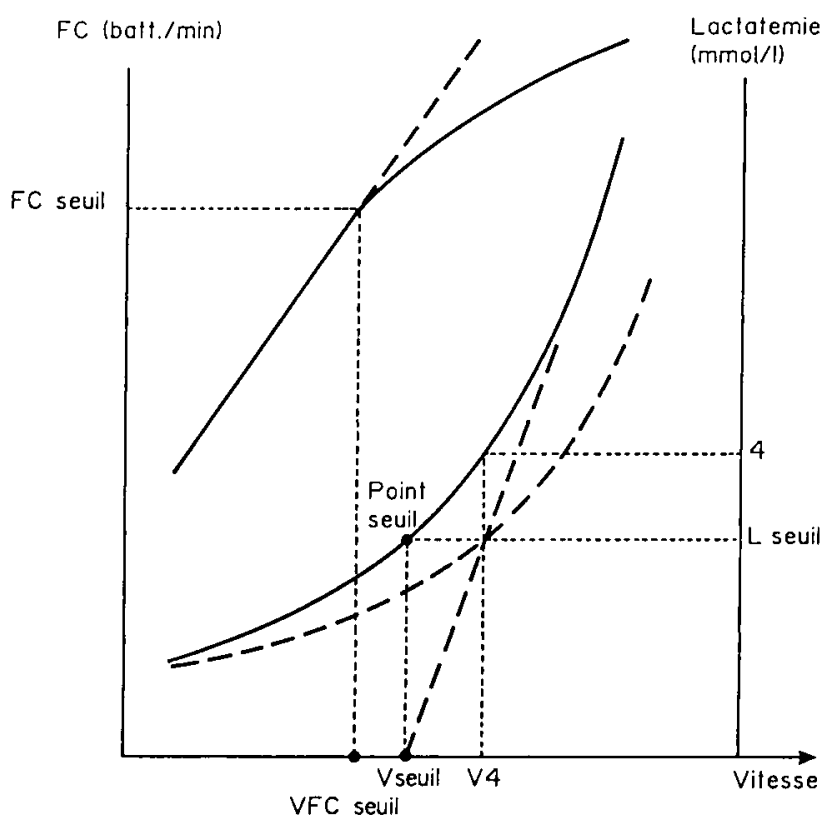

Fig. 1. Détermination graphique des seuils. 
chez l'homme (Heck et al., 1985), sert à la conduite de l'entraînement en endurance chez celui-ci.

Chez le cheval, la plupart des auteurs ont utilisé le paramètre $V_{4}$ (vitesse induisant une lactatémie de $4 \mathrm{mmol}^{-1} \mathrm{I}^{-1}$ ) pour évaluer l'effet de l'entraînement (Wilson et al., 1983), l'état de forme (Bayly, 1986; Sloet Van Oldruitenborgh-Oosterbaan et al., 1987) ou l'intérêt d'une ration alimentaire adaptée à l'effort long (Wolter et al., 1987), sans préjuger de la réalité de cette valeur comme seuil anaérobie chez le cheval. Saibene et al. (1985) et Ceretelli (1987) préfèrent utiliser la vitesse d'accumulation du lactate $(\Delta L a / \Delta t)$ énoncée par Margaria et al. (1963). Blanc et al. (1987) comme Michaux (1988) estiment le useuil d'accumulation du lactate" d'après la position du point de rupture de la linéarité dans la relation lactatémie vs vitesse.

A l'instigation de Persson (1983), le paramètre $V_{200}$ (vitesse induisant une fréquence cardiaque de $200 \mathrm{batt} / \mathrm{min}^{-1}$ ) qui ne correspond aucunement à un seuil mais reflète le fonctionnement cardiaque lors d'un effort situé au-delà du seuil anaérobie, s'avère d'un abord aisé pour la conduite de l'entraînement sur le terrain. Ce paramètre $V_{200}$ a été repris par de nombreuses équipes (Wilson et al., 1983; Bayly, 1986; Evans \& Rose, 1987; Valette \& Wolter, 1987). Une estimation du seuil cardiaque anaérobie (Conconi et al., 1982; Persson, 1983; Rose \& Evans, 1987) pour- rait se situer au point de déflexion (Fig. 1) de la relation fréquence cardiaque vs vitesse.

Le but du présent travail a été de déterminer les seuils lactique et cardiaque chez le poney, leurs particularités individuelles et leurs évolutions en fonction du type d'entraînement.

\section{MATÉRIEL ET MÉTHODES}

Six poneys de conformations différentes ( 140 à $220 \mathrm{~kg}$ ) sont entraînés en aérobiose, à $65 \%$ de leur fréquence cardiaque maximale (130-140 batt.min-1) 3 fois par semaine pendant 6 mois; puis ils sont mis au pré pendant 2 mois, avant de reprendre un entraînement, essentiellement en anaérobiose (200 batt.min ${ }^{-1}$ ), 2 fois par semaine pendant 5 semaines. Les épreuves de contrôle ont lieu sur tapis roulant après $10 \mathrm{~min}$ d'échauffement au petit trot à la vitesse de 1,9$2,5 \mathrm{~m} / \mathrm{s}$. La fréquence cardiaque $(F C$ ) est enregistrée en continu au moyen d'un cardiofréquencemètre (Horse Tester). (Fig. 2).

Le protocole consiste en 2 séries d'épreuves

\section{Epreuves tests par paliers}

Des épreuves test $\mathrm{ET}_{1}, \mathrm{ET}_{2}, \mathrm{ET}_{3}$, comportant 6 paliers de vitesses croissantes (de 2 à $7 \mathrm{~m} / \mathrm{s}$ ), adaptées à chaque animal afin que les 2 derniers paliers s'effectuent à une fréquence car-

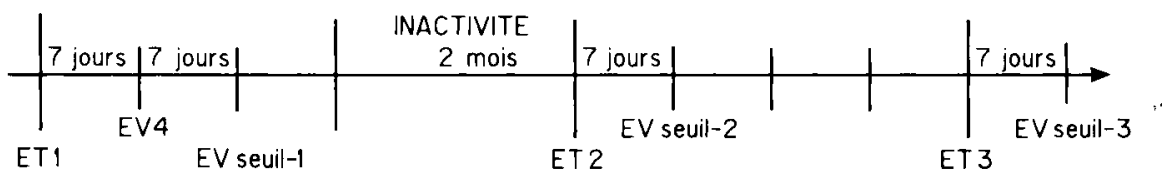

Fig. 2. Chronologie des épreuves. 
diaque supérieure à 200 batt.min $^{-1}$. Chaque palier d'effort dure $3 \mathrm{~min}$ avec un intervalle de repos de $2 \mathrm{~min}$ au cours duquel sont effectuées les prises de sang.

L'étude des relations entre vitesse ( $V$, lactatémie $\left(L_{\mathrm{a}}\right)$ et fréquence cardiaque (FC) définit un certain nombre de critères physiologiques qui évaluent l'aptitude à l'effort (Valette \& Wolter, 1988), en particulier $V_{4}$.

Pour chaque individu, les lactatémies et les fréquences cardiaques obtenues aux paliers successifs permettent de construire, par interpolation les courbes $L a$ et $F C$ vs vitesse.

La traduction graphique (Fig. 1), réalisée par la méthode des Splines cubiques (Dony, 1986) conduit à estimer les caractéristiques du seuil lactique au-delà duquel le métabolisme est essentiellement anaérobie avec accumulation d'acide lactique. L'extension de la branche supérieure de l'exponentielle sur l'axe des abscisses, indique la vitesse seuil $\left(V_{\text {seuil }}\right)$. Le point seuil de la courbe (point de courbure maximale) projeté sur l'axe des ordonnées détermine la lactatémie seuil ( $\left.L_{\text {seuil }}\right)$. De même, la fréquence seuil ( $\left.F C_{\text {seuil }}\right)$ est déterminée graphiquement au point de déflexion de la courbe FC vs vitesse (Conconi, 1982).

\section{Epreuves à vitesse constante}

En alternance avec les épreuves par paliers, des épreuves sont effectuées à vitesses constantes (Fig. 2) différentes pour chaque poney, appelées $E V_{4}\left(10 \mathrm{~min}\right.$ à la vitesse $V_{4}$ déterminée lors de $E T 1$ ) ou $E V_{\text {seull }}$ (2 phases successives de $10 \mathrm{~min}$ aux vitesses seuil déterminées par l'épreuve test immédiatement précédente), ponctuées par un prélèvement sanguin.

Les échantillons de sang obtenus lors des différentes épreuves servent au dosage de

Tableau I. Résultats des épreuves test, à paliers de vitesses croissantes.

\begin{tabular}{|c|c|c|c|c|c|c|c|}
\hline Poney & 1 & 2 & 3 & 4 & 5 & 6 & $m \pm t s / V n$ \\
\hline \multicolumn{8}{|c|}{$\begin{array}{l}\text { Epreuve test } \\
\text { ET. }\end{array}$} \\
\hline$v_{4}$ & 6,2 & 5,55 & 5,0 & 5,3 & 5,1 & 5,3 & $5,4 \pm 0,40$ \\
\hline$V_{\text {seuil }}^{4}$ & 5,1 & 4,9 & 4,5 & 4,6 & 4,3 & 4,2 & $4,6 \pm 0,3$ \\
\hline$L_{\text {sevil }}^{\text {sell }}$ & 2,1 & 2,3 & 2,9 & 2,4 & 2,6 & 2,1 & $2,4 \pm 0,3$ \\
\hline$F C_{\text {seuil }}$ & 152 & 176 & 185 & 166 & 174 & 169 & $170 \pm 11$ \\
\hline \multicolumn{8}{|l|}{$\mathrm{ET}_{2}$} \\
\hline$V_{4}{ }^{4}$ & 5,7 & 4,8 & 4,55 & 5,35 & 3,55 & 3,45 & $4,55 \pm 0,85$ \\
\hline$V_{\text {seuil }}$ & 4,6 & 4,8 & 4,2 & 4,0 & 3,5 & 4,3 & $4,2 \pm 0,4$ \\
\hline$L_{\text {seuil }}^{\text {seul }}$ & 2,1 & 4,0 & 4,0 & 2,2 & 3,6 & 3,5 & $3,2 \pm 0,8$ \\
\hline$F C_{\text {seuil }}$ & 134 & 153 & 131 & 121 & 135 & 166 & $140 \pm 16$ \\
\hline \multicolumn{8}{|l|}{$\mathrm{ET}_{3}$} \\
\hline$V_{4}$ & 4,95 & 4,3 & 3,55 & 4,45 & 3,75 & 3,35 & $4,05 \pm 0,55$ \\
\hline$V_{\text {seuil }}$ & 4,6 & 4,6 & 4,3 & 4,8 & 3,6 & 3,7 & $4,3 \pm 0,5$ \\
\hline$L_{\text {seuil }}$ & 3,0 & 3,3 & 3,5 & 3,4 & 3,9 & 3,3 & $3,4 \pm 0,3$ \\
\hline$F C_{\text {seuil }}$ & 180 & 180 & 165 & 185 & 190 & 175 & $179 \pm 9$ \\
\hline
\end{tabular}

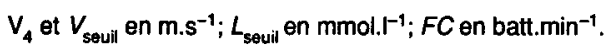


Tableau II. Lactatémies $\left(m m o l . I^{-1}\right)$ obtenues pour l'épreuve, à vitesse constante $\mathrm{V}_{4}\left(\mathrm{EV}_{4}\right)$. Blood lactate levels at constant speed $\mathrm{V}_{4}$ test.

\begin{tabular}{llllllll}
\hline Poney & 1 & 2 & 3 & 4 & 5 & 6 & $m \pm t \mathrm{~s} / \mathrm{Nn}$ \\
Lactatémie & 8,25 & 10,1 & 5,9 & 8,2 & 7,7 & 8,85 & $8,2 \pm 1,4$ \\
\hline
\end{tabular}

l'acide lactique (Analox). Les résultats sont traités par le test de Student et l'étude du coefficient de corrélation (Schwartz, 1969).

\section{RÉSULTATS}

Les données obtenues lors des 3 épreuves test (ET) sont indiquées dans le Tableau I. Le Tableau II rapporte les résultats relatifs aux lactatémies lors de l'épreuve $\mathrm{EV}_{4}\left(10 \min\right.$ à $\left.V_{4}\right)$. Le Tableau III récapitule les lactatémies : (a) en mmol..$^{-1}$ et les fréquences cardiaques; (b) en batt. min $^{-1}$ constatées à chaque phase lors des 3 épreuves $\mathrm{EV}_{\text {seuil }}$ (2 séquences de 10 $\min$ à $\left.V_{\text {seuil }}\right)$.

$V_{4}$ et $V_{\text {seuil }}$ se révèlent corrélées ( $r_{16 \text { dal }}$ $=0,66$; s à $1 \%$ ) et se situent pour les 2 premières épreuves test à des valeurs différentes $\left(t_{11 d d l}=3,2 ; s\right.$ à $\left.1 \%\right)$. Les valeurs $V_{\text {seuil }}$ sont inférieures aux valeurs $V_{4}(\mathrm{Ta}$ bleau I). Les particularités de l'entraine-

Tableau III. Lactatémies (a, mmol. $\left.\right|^{-1}$ ) et fréquences cardiaques (b, batt.min ${ }^{-1}$ ) obtenues pour les épreuves à vitesse constante $V_{\text {seuil }}$.

\begin{tabular}{|c|c|c|c|c|c|c|c|c|}
\hline Poney & & 1 & 2 & 3 & 4 & 5 & 6 & $m \pm t s / V n$ \\
\hline $\begin{array}{l}E V_{\text {seuil }}-1 \\
\text { phase } 1\end{array}$ & $\begin{array}{l}1 \\
-a \\
-b\end{array}$ & $\begin{array}{l}1,9 \\
164\end{array}$ & $\begin{array}{l}1,2 \\
172\end{array}$ & $\begin{array}{l}3,7 \\
186\end{array}$ & $\begin{array}{l}1,7 \\
192\end{array}$ & $\begin{array}{l}1,4 \\
182\end{array}$ & $\begin{array}{l}1,2 \\
155\end{array}$ & $\begin{array}{l}1,9 \pm 0,9 \\
175 \pm 14\end{array}$ \\
\hline phase 2 & $\begin{array}{l}-a \\
-b\end{array}$ & $\begin{array}{l}2,4 \\
159\end{array}$ & $\begin{array}{l}1,3 \\
174\end{array}$ & $\begin{array}{l}3,8 \\
189\end{array}$ & $\begin{array}{l}1,9 \\
193\end{array}$ & $\begin{array}{l}2,1 \\
179\end{array}$ & $\begin{array}{l}1,3 \\
170\end{array}$ & $\begin{array}{l}2,1 \pm 0,9 \\
177 \pm 13\end{array}$ \\
\hline $\begin{array}{l}E V_{\text {seuil }}-2 \\
\text { phase } 1\end{array}$ & $\begin{array}{l}2 \\
-a \\
-b\end{array}$ & $\begin{array}{l}2,1 \\
149\end{array}$ & $\begin{array}{l}4,0 \\
178\end{array}$ & $\begin{array}{l}4,0 \\
142\end{array}$ & $\begin{array}{l}1,8 \\
124\end{array}$ & $\begin{array}{l}2,2 \\
134\end{array}$ & $\begin{array}{l}3,5 \\
155\end{array}$ & $\begin{array}{l}2,9 \pm 1,0 \\
147 \pm 19\end{array}$ \\
\hline phase 2 & $\begin{array}{l}-a \\
-b\end{array}$ & $\begin{array}{l}3,1 \\
153\end{array}$ & $\begin{array}{l}3,9 \\
170\end{array}$ & $\begin{array}{l}4,7 \\
135\end{array}$ & $\begin{array}{l}2,2 \\
135\end{array}$ & $\begin{array}{l}2,4 \\
138\end{array}$ & $\begin{array}{l}3,3 \\
143\end{array}$ & $\begin{array}{l}3,3 \pm 0,9 \\
146 \pm 14\end{array}$ \\
\hline $\begin{array}{l}\mathrm{EV}_{\text {seuil }}-3 \\
\text { phase } 1\end{array}$ & $\begin{array}{l}3 \\
-a \\
-b\end{array}$ & $\begin{array}{l}3,4 \\
160\end{array}$ & $\begin{array}{l}3,7 \\
170\end{array}$ & $\begin{array}{l}4,0 \\
191\end{array}$ & $\begin{array}{l}3,2 \\
171\end{array}$ & $\begin{array}{l}4,2 \\
193\end{array}$ & $\begin{array}{l}2,7 \\
163\end{array}$ & $\begin{array}{l}3,5 \pm 0,6 \\
175 \pm 14\end{array}$ \\
\hline phase 2 & $\begin{array}{l}-a \\
-b\end{array}$ & $\begin{array}{l}3,4 \\
158\end{array}$ & $\begin{array}{l}4,3 \\
179\end{array}$ & $\begin{array}{l}3,6 \\
174\end{array}$ & $\begin{array}{l}3,1 \\
175\end{array}$ & $\begin{array}{l}3,9 \\
168\end{array}$ & $\begin{array}{l}3,0 \\
170\end{array}$ & $\begin{array}{l}3,6 \pm 0,5 \\
171 \pm 7\end{array}$ \\
\hline
\end{tabular}


ment avant $\mathrm{ET}_{3}$ (travail anaérobie) tendent à inverser les priorités entre $V_{\text {seuil }}$ et $V_{4}$ (Tableau I).

Les valeurs $L_{\text {seuil }}$ varient de 2,4 à 3,6 mmol. $1^{-1}$ (Tableau I) selon que l'entraînement privilégie les processus aérobies $\left(E T_{1}\right)$ ou anaérobies $\left(E T_{3}\right)$, tandis que $V_{4}$ passe de 5,40 à $4,05 \mathrm{~m} / \mathrm{s}$. Les valeurs $V_{4}$ et $L_{\text {seuil }}$ sont d'ailleurs corrélées négativement $\left(r_{16 \mathrm{ddl}}=-0,78\right.$; s à 1 p. 100); par contre, $V_{\text {seuil }}$ n'est corrélée ni avec $L_{\text {seuil }}$ $(r=-0,36)$ ni avec $F C_{\text {seuil }}(r=0,14)$.

Les lactatémies observées lors de $\mathrm{EV}_{4}$ (Tableau II) sont très nettement au-dessus des valeurs obtenues après 3 min à cette vitesse lors de $E T_{1}$.

Les lactatémies augmentent légèrement $\left(t_{17 d d l}=2,35 ; s\right.$ à 5 p. 100) entre les 2 paliers de $10 \mathrm{~min}$ à $V_{\text {seuil }}$ (Tableau III) tandis que les fréquences cardiaques du fait de leur plus grande dispersion restent stables $\left(t_{17 \mathrm{ddl}}=0,44 ; \mathrm{ns}\right)$. Malgré tout, les valeurs finales des lactatémies observées après $20 \mathrm{~min}$ d'effort ne sont pas différentes des seuils estimés ( $t_{17 \mathrm{ddl}}=0,17$; ns). Il en est de même pour les fréquences cardiaques $\left(t_{17 \mathrm{ddl}}=0,41 ; \mathrm{ns}\right)$.

\section{DISCUSSION}

La valeur 4 mmol.l-1 ne semble pas correspondre, chez le poney, à un seuil, c'està-dire un état stable où la production d'acide lactique par certaines fibres musculaires égale la consommation de ce métabolite par d'autres fibres ou par le foie. En effet, il existe une discordance entre les lactatémies obtenues à la vitesse $V_{4}$ selon que la durée de l'exercice est de 3 $\min \left(E T_{1}\right)$ ou de $10 \mathrm{~min}\left(E V_{4}\right)$. Cette influence de la durée d'effort sur la lactatémie obtenue à la vitesse $V_{4}$ contredit la no- tion même de seuil pour la valeur $4 \mathrm{mmol}$ qui devrait rester stable pendant une longue durée.

Par contre, les vitesses seuils déterminées graphiquement semblent bien estimer cet état stable puisque l'on observe peu de variations, que l'exercice dure 3,10 ou $20 \mathrm{~min}$. Les fluctuations de la lactatémie seuil entre les individus montrent que le point de plus grande courbure de la relation lactatémie vs vitesse n'est pas une constante biologique comme le laisse supposer la référence $4 \mathrm{mmol}^{-1}{ }^{-1}$ peu spécifique, mais une donnée individuelle obtenue dans des conditions particulières. En effet, les caractéristiques de ce point ( $V_{\text {seuil, }}$ $\left.L_{\text {seuil }}\right)$ peuvent être modifiées en fonction de l'individu, des conditions de course et des modalités d'entraînement. Par rapport à une période d'activité moyenne (mise au pré), un entraînement en aérobie (endurance) abaisse la lactatémie seuil, tout en augmentant ( $V_{\text {seuil }}$ et $V_{4}$ alors qu'un entraînement en anaérobie (sprint) élève la lactatémie seuil tout en diminuant les valeurs $V_{\text {seuil }}$ et $V_{4}$. Dans ce dernier cas, contrairement à l'entraînement en endurance, $V_{\text {seuil }}$ et $V_{4}$ sont presque confondues (Tableau I). Ces valeurs seuils dépendent donc non seulement de l'individu, mais aussi de leur niveau de préparation.

Malgré tout, le suivi de l'indice $V_{4}$ ou mieux son corrolaire $\mathrm{FF}_{4}$ (fréquence des foulées induisant une lactatémie de 4 mmol.1-1) peut suffire pour suivre la progression dans une discipline donnée, la courbe lactatémie vs vitesse (Fig. 1) se déplaçant vers la droite avec l'entraînement (Valette \& Wolter, 1988), avec amélioration de $V_{\text {seuil }}$ et $V_{4}$, que la lactatémie seuil soit basse (endurance) ou élevée (sprint). La connaissance exacte de la lactatémie seuil d'un individu se justifie pour sa sélection vers un type particulier d'entraînement.

La $F C_{\text {seuil }}$ estimée par la position du point de déflexion semble mieux corres- 
pondre à la notion de seuil que le critère $\mathrm{V}_{200}$. Par ailleurs, la détermination du seuil cardiaque au moyen d'un cardiofréquencemètre, demande moins de technicité que le dosage du lactate sanguin et peut permettre de déterminer si l'animal est en forme ( $F C_{\text {seuil }}$ élevée) ou non ( $F C_{\text {seuil }}$ basse). En ce sens, le paramètre $V_{\text {seuil }}$ $(F C)$ obtenu d'après la relation $F C$ vs vitesse (vitesse induisant la fréquence cardiaque seuil) serait intéressant à considérer (Fig. 1) pour les tests d'effort sur piste.

\section{RÉFÉRENCES}

Barrey E., Valette J.P. \& Wolter R. (1989) Etude multifactorielle de l'aptitude à l'effort. Ann. Zootech. 38, 127-139

Bayly W. (1986) From some practical aspects of performance in the horse. Equine Sports Med. News 5, 70-72

Blanc P., Antonioni M.T., Villeneuve P., Virot P., Dalmay F. \& Nicolas A. (1987) Aptitude physique du cheval : dosage du lactate au cours d'épreuves d'effort triangulaires. Congrès de Chirurgie Equine et Médecine des Sports Equestres, 25-28 mars, Genève, 290-292

Brooks G.A. (1985) Anaerobic threshold: review of the concept and directions for future research. Med. Sci. Sports Exerc. 17, 22-31

Ceretelli P. (1987) Blood lactate and exercise in the horse. Physiological significance and practical implications. Congrès de Chirurgie Equine et Médecine des Sports Equestres, 25-28 mars, Genève, 269

Conconi F., Ferrari M., Droghetti P. \& Condeca L. (1982) Determination of the anaerobic threshold by a non-invasive field test in turners. $J$. Appl. Physiol. 52, 869-873

Di Prampero P. (1986) Evolution de la lactatémie au cours de l'effort en fonction du temps. V'^ Séminaire de Bioénergétique, INSEP, Paris, 20-21 mars, 9-11

Dony R. (1986) Calcul des Parties Cachées : Approximation des courbes par la Méthode de Bézier et de B-splines. Collection Méthode et Programme, Masson, Paris, 237 pp.
Evans D.L. \& Rose R.J. (1987) Maximum oxygen uptake in race horses: changes with training state and prediction from submaximal cardiorespiratory measurements. In : Equine Exercise Physiology, vol. 2, Proc. 2nd International Conference on Equine Exercise Physiology. ICEEP Publications, Davis, CA, USA, 52-67

Heck H., Mader A., Hess S., Mucke S., Muller R. \& Hollmann W. (1985) Justification of the 4 mmol// lactate threshold. Int. J. Sports Med. 6, 117-130

Hollmann W. (1985) Historical remarks on the development of the aerobic-anaerobic threshold up to 1966. Int. J. Sports Med. 6, 109-116

Keul J., Simon G. \& Berg A., Dickhuth H., Goertler L. \& Kuber R. (1979) Bestimmung des individuellen anaeroben Schwelle zur Leitungbewertung und Trainingestaltung. Deutsch. $Z$. Sportmed. 30, 212-218

Kindermann W., Simon G., Keul J. (1979) The significance of the aerobic-anaerobic transition for determination of work load intensities during endurance training. Eur. J. Appl. Physiol. 42, 2534

Mader A., Liesen H., Heck H., Philippi H., Rost R., Schurch P.M. \& Hollmann W. (1976) Beurteilung der sportartspezifischen Ausdauer leistungsfähigkeit im Labor. Sportartz. Sportmed. $27,80-88$

Margaria R., Aghemo P. \& Sassi G. (1963) Lactic acid production in supramaximal exercise. Pflügers Arch. 326, 152-161

Michaux J.M. (1988) Determination des seuils anaérobie chez le cheval d'endurance. Mémoire de Recherche pour le Concours d'Agrégation Vétérinaire, ENV de Lyon, 80 pp.

Persson S.G.B. (1983) Evaluation of exercise tolerance and fitness in the performance horse. In: Equine Exercise Physiology, Granta, Cambridge, 441-457

Rieu M., Barrault D., Duvallet A. \& Ferry A. (1986) La zone de transition "aérobieanaérobie". VP séminaire de Bioénergétique, Revue EPS, Paris, 59 pp.

Rose R.J. \& Evans D.L. (1987) Cardiovascular and respiratory function in the athletic horse. In : Equine Exercise Physiology, vol. 2, Proc. 2nd International Conference on Equine Exercise Physiology. ICEEP Publications, Davis, CA, USA, 124 
Saibene F., Cortili G., Gavazzi P., Sala A., Faina M. \& Sardella F. (1985) Maximal anaerobic (lactic) capacity and power of the horse. Equine Vet. J. 17, 130-132

Schwartz D. (1969) Méthodes statistiques à I'Usage des Médecins et des Biologistes. Coll. Médecine et Sciences, Flammarion, Paris, $318 \mathrm{pp}$.

Sjodin B. \& Jacob 1. (1981) Onset of blood lactate accumulation and marathon running performance. Int. J. Sports Med. 2, 23-26

Sloet Van Oldruitenborgh-Oosterbaan M.M., Wensing T. \& Breukink H.J. (1987) Standardized exercise test on a track to evaluate fitness and training of saddle horses. In: Equine Exercise Physiology, vol. 2, Proc. 2nd International Conference on Equine Exercise Physiology. ICEEP Publications, Davis, CA, USA, 68-76

Valette J.P. \& Wolter R. (1987) Lactate et rythme cardiaque : critères d'aptitude sportive chez le poney. Congrès de Chirurgie Equine et
Médecine des Sports Equestres, Genève, 2528 mars, 270-276

Valette J.P. \& Wolter R. (1988) intérêt des mesures de lactatémie et de fréquence cardiaque comme critères d'aptitudes sportives. CEREOPA, $14^{\theta}$ journée d'étude, 9 mars, 1-16

Wasserman K. \& Mcllroy M.B. (1964) Detecting the threshold of anaerobic metabolism in cardiac patients during exercise. Am. J. Cardiol. 14, 844-852

Wilson R.G., Isler R.B. \& Thornton J.R. (1983) Heart rate, lactic acid production and speed during a standardized exercise test in standardbred horses. In: Equine Exercise Physiology, Granta, Cambridge, 487-496

Wolter R., Valette J.P., Ezzaouia M., Henry N., Grandjean R. \& Jouglin M. (1987) Influence d'une surcharge en minéraux et/ou vitamines sur les aptitudes sportives du poney. CEREO$\mathrm{PA}, 13^{\circ}$ journée d'étude, 11 mars, 111-121 